\section{LA MODERNIZACIÓN DE LAS FUERZAS ARMADAS}

\author{
Eduardo Serra \\ Ex Ministro de Defensa \\ eserrar@esya.es
}

\section{MODERNISATION OF THE ARMED FORCES}

Cómo citar este artículo/Citation: Serra, E. (2014). "La modernización de las Fuerzas Armadas". Arbor, 190 (765): a098. doi: http://dx.doi.org/10.3989/arbor.2014.765n1005
Copyright: @ 2014 CSIC. Este es un artículo de acceso abierto distribuido bajo los términos de la licencia Creative Commons Attribution-Non Commercial (by-nc) Spain 3.0.

Recibido: 5 junio 2012. Aceptado: 2 diciembre 2013.

RESUMEN: El artículo realiza un breve repaso por la historia de las Fuerzas Armadas españolas destacando la importancia de la institución militar y su papel en la Historia contemporánea española. Desde de la muerte de Franco se intenta por encauzar a los mandos de las Fuerzas Armadas en la senda democrática y constitucional, siendo entonces crucial el papel del General Gutiérrez Mellado. A partir de 1981, se toman dos importantes medidas en la esfera militar: la llamada causa $2 / 81$, para el enjuiciamiento de los responsables de la intentona golpista y la solicitud de ingreso de España en la Alianza Atlántica, paso muy importante que determinará el futuro del país y permitió la modernización de las Fuerzas Armadas españolas, equiparándolas a las del mundo occidental. Tras la victoria socialista de 1982, fue básico el giro del PSOE, que transformando su antiamericanismo inicial con habilidad, completó el ingreso de España en la Alianza Atlántica tras el referéndum de 1986, y modificó profundamente las Fuerzas Armadas, eliminando las tensiones militares. La última etapa del proceso de modernización fue la protagonizada por el gobierno de Aznar: destacan la profesionalización de las Fuerzas Armadas y la integración de España en la estructura militar de la Alianza Atlántica.

PALABRAS CLAVE: España; Ejército; Fuerzas Armadas; Modernización; General Gutiérrez Mellado; Rey.
ABSTRACT: This article briefly reviews the recent history of the Spanish Armed Forces, stressing the importance of the military institution and its role in contemporary Spanish history. Following Franco's death there was an attempt to bring the command of the Armed Forces under democratic control within the constitution, a process in which General Gutiérrez Mellado played a crucial role. Since 1981, two important measures have been taken in the military sphere: "case $2 / 81$ ", prosecuting the perpetrators of the attempted coup in 1981, and Spain's application to join the Atlantic Alliance (NATO), a very important step in shaping the country's future and driving the modernisation of the Spanish Armed Forces, putting them on an equal footing with their peers elsewhere in the Western world. After the socialist victory in 1982, the PSOE government changed direction, skilfully transforming its initial anti-Americanism, obtaining Spain's membership of NATO following the 1986 referendum, and profoundly changing the Armed Forces, thus eliminating military tensions. The last stage of the modernisation process was led by Aznar's government, with the professionalisation of the Armed Forces and the integration of Spain in NATO's military structure.

KEYWORDS: Spain; Army; Armed Forces; Modernisation; General Gutiérrez Mellado; King. 
La razón de ser de las Fuerzas Armadas, de España y de cualquier país, es la defensa de los valores constitucionales y de los intereses nacionales frente al enemigo. Se sobreentiende que el enemigo es, por definición, del exterior. España es el primer país europeo que emerge como estado nacional moderno en las postrimerías del siglo XV organizándose desde muy pronto sus ejércitos (las primeras Ordenanzas Militares son de 1505) y tiene desde entonces, por no hablar de antes, probablemente el mejor historial militar de Europa. Flandes e Italia son los primeros escenarios de una nueva manera de combatir que se revela como extremadamente eficaz y que asombra a los demás ejércitos. En Rocroi (1643), sin embargo, este prestigio y con él el de España, empieza a declinar iniciándose entonces una larguísima decadencia que, con algunos conatos de recuperación, ha llegado a nuestros días. España, que había enseñoreado el mundo, se cierra sobre sí misma, herida y dolida, enzarzándose en un sinfín de contiendas civiles y guerras coloniales que arruinan el país y minan su confianza en sí misma. Antonio Machado, aplicándolo a Castilla, describe probablemente mejor que nadie este estado de ánimo:

Castilla miserable, ayer dominadora,

envuelta en sus andrajos desprecia cuanto ignora

No es extraño que en esta tesitura el ejército fuera utilizado muchas veces para dar giros más o menos radicales a la situación política del momento (Seco Serrano, 1984); todo ello culmina con la más espantosa guerra civil de 1936-1939, precedida por una serie de campañas (las guerras de África) en las que nuestro ejército no sale demasiado bien parado sin perjuicio de muy notorios heroísmos singulares y colectivos.

Tras cuarenta años de dictadura, en 1975, se inicia un periodo que, bajo el frontispicio de la reconciliación nacional, parece abordar el futuro con energías renovadas. En efecto, la Constitución de 1978, la primera inspirada en el consenso, inaugura así un periodo de paz, libertad y prosperidad (esta última iniciada ya a partir del plan de estabilización de 1959) que parece haber encarrilado definitivamente la incardinación de España en la Modernidad. Este periodo de Monarquía Constitucional ha protagonizado el mayor cambio acaecido en Europa en la segunda mitad del siglo XX, hasta la caída del muro de Berlín en 1989, periodo conocido como "la transición democrática". En efecto, en un lapso de tiempo extraordinariamente breve, España pasa de ser un país aislado, pobre, agrícola, rural y sometido a una dictadura, a ser un país plenamente inserto en el contexto internacional, rico, urbano, industrial y de servicios y disfrutar de una democracia que, aunque ciertamente perfectible, debe asegurar la estabilidad a largo plazo.

Recuerdo al profesor Raymond Carr que, en los primeros años 80 me mostraba su escepticismo de que España fuera capaz de superar sus dos grandes problemas seculares, el regional y el militar. Eran los tiempos, recuérdese, de especial virulencia terrorista de ETA y de inquietudes militares que habían aflorado en el intento del 23 de febrero de 1981.

Sin embargo, poco tiempo después, en 1986, en una recepción en la Embajada española en Londres me mostraba su estupefacción ante la actitud derrotista de los españoles cuando a su juicio habíamos realizado la increíble hazaña de superar esos dos tradicionales problemas, especialmente -subrayaba- el militar; del otro, del problema regional, decía, "le han dado ustedes una solución, o mejor una salida, la de las Comunidades Autónomas", de la que decía no sabía si era la mejor, pero al menos era una salida. Se entendía por "problema militar" la incapacidad del ejército español para aceptar un régimen democrático con todas sus consecuencias; pero el citado intento de golpe de Estado no solo había fracasado (porque la inmensa mayoría de los militares no lo apoyaron, permaneciendo leales a la Constitución y sus Instituciones) sino que habría actuado como una especie de vacuna para futuras e hipotéticas intentonas; ello unido al cambio socioeconómico operado en España, que habría extendido extraordinariamente el volumen de la clase media (aproximadamente de un $17 \%$ en la década de los 30 a un $75 \%$ al final de la Dictadura) y, con ello, llevado la moderación a las urnas, hacía impensable un nuevo pronunciamiento militar.

Sirva esta larga introducción para enmarcar la importancia de la institución militar y su papel en la Historia contemporánea española para así entender en su cabal medida la trascendencia del proceso de su modernización. La modernización de nuestras Fuerzas Armadas suponía pues situarlas claramente bajo el poder civil, como sucedía - y sucede - en los países desarrollados y ceñir su actuación a las funciones estrictamente militares, vinculadas por tanto a la Política Exterior y des-responsabilizándolas de modo claro y tajante de las vicisitudes políticas internas, cualesquiera que estas fueran. El singular proceso de modernización de las Fuerzas Armadas no podría entenderse además si no se entiende que los militares tienen, en mucha mayor medida que otros grupos so- 
ciales, cualidades tales como el espíritu de servicio, el amor a la patria y la fe en el futuro de la nación, y también una cierta renuncia a las propias ambiciones en aras de las ambiciones colectivas, lo que se podría llamar capacidad de sacrificio.

Así las cosas, en 1975 nos encontramos con unas Fuerzas Armadas claramente sobredimensionadas (cerca de medio millón de personas), pobremente dotadas de un material escaso y en su mayoría obsoleto, y ejerciendo multitud de funciones que en otros países de nuestro entorno eran desarrolladas por civiles. Ello es consecuencia lógica de que, por aquel entonces, España era todavía un país relativamente aislado, pues, aunque desde el punto de vista social y económico estábamos totalmente abiertos (turistas, emigrantes, balanza comercial, etc. etc.) sin embargo desde el punto de vista político todavía estábamos notablemente aislados (Unión Europea, Alianza Atlántica, etc.); por lo que, carentes prácticamente de razón de ser exterior más allá de nuestras posesiones en África, los militares se ocupaban de funciones que pudieran no corresponder a las Fuerzas Armadas, singularmente el orden público.

Además de todo ello, la mayor parte de nuestros militares, como muchos otros españoles de la época, sentían como propio el régimen de Franco (un militar) que había vencido en la Guerra Civil salvando así a su juicio a España de la tiranía comunista; por lo que, a la muerte de aquél, sentían, como todos, la incertidumbre sobre el futuro, pero además, el temor a que se desmantelara un régimen que en su sentir mayoritario, había sido extraordinariamente positivo para España. La única garantía para ellos la daba el Rey Juan Carlos (también militar); su preocupación principal era la ruptura de la unidad de la patria, por lo que les produjo una gran tranquilidad el artículo 8 응 de la Constitución de 1978, que responsabiliza a las Fuerzas Armadas, entre otras, de la misión de defender la integridad de España; recelosos también de las intromisiones de los partidos políticos, les tranquilizó que el texto constitucional otorgara al Rey la Jefatura Suprema de las Fuerzas Armadas.

Por todo lo que antecede, la primera etapa de nuestra vida política tras la muerte de Franco (1975-1981) está presidida por las tensiones militares causadas por las primeras medidas de muy diversa índole adoptadas para la normalización democrática de nuestras instituciones. La más importante de todas, la que más tensiones creó, fue el reconocimiento del Partido Comunista de España en la Semana Santa de 1977, pero también las manifestaciones separatistas de distinto signo que incluían retirada, e incluso quema de banderas españolas, así como numerosas expresiones en contra de la unidad de España. Esta etapa tiene un personaje crucial: el General Gutiérrez Mellado, quien emprende la tarea de introducir a los mandos de las Fuerzas Armadas en la senda democrática y constitucional. En efecto, el General Gutiérrez Mellado, secundado poco después por Agustín Rodríguez Sahagún (en 1979, primer Ministro de Defensa civil desde los tiempos de la Segunda República), acomete las primeras y por tanto más difíciles reformas que comprometen el estatus de relativa autonomía así como las ideas políticas e inquietudes de la mayoría de nuestros militares: así la Ley Orgánica de Criterios Básicos de la Defensa Nacional, las nuevas Reales Ordenanzas para las Fuerzas Armadas, la creación del Ministerio de Defensa en 1977, sustituyendo a los tres antiguos ministerios militares y dentro de él, los nuevos Servicios de Inteligencia (CESID) sobre la base del antiguo SECED, pero excluyendo las competencias en materia de política interior, exceptuando la involución y el terrorismo, etc. Todo ello en un clima de extendida incomprensión por parte de sus compañeros de armas, algunos de los cuales realizan actos de ostentosa indisciplina militar que exigen la debida reprensión. La aguda crisis económica, las tensiones de las recién creadas Comunidades Autónomas y, sobre todo, el número y gravedad de los actos terroristas realizados por ETA, conducen a un clima irrespirable que estalla en la intentona referida del 23 de febrero 1981.

Aunque todavía nos falta distancia histórica, a mi juicio no se ha reconocido como merece la labor del General Gutiérrez Mellado: él y su pequeño equipo fueron capaces no solo de hacer frente a la incomprensión y rechazo de muchos militares, sino que sentaron las bases de lo que son hoy las Fuerzas Armadas de una democracia plenamente integrada en el mundo occidental. La imagen del General Gutiérrez Mellado intentando contener en el hemiciclo del Congreso de los Diputados el asalto golpista del teniente Coronel Tejero, quedará para siempre como la mejor representación de la definitiva victoria del poder legítimo en la monarquía del Rey Juan Carlos, quien por cierto jugó un papel trascendental en este crucial episodio: el hecho de que La Constitución otorgase al Rey la Jefatura Superior de las Fuerzas Armadas unido al automatismo de la disciplina militar, hizo que la firme posición del Rey en favor del poder civil mostrada en directo por las cámaras de la televisión, fuera la causa principal de la derrota de los conjurados. En virtud de ello, el Rey consolidó de modo indeleble su figura, se ganó el reconocimiento de propios y extraños e hizo 
ver, quizás por primera vez, el acierto de haber escogido la monarquía como forma de gobierno para la mejor garantía del poder civil.

La segunda etapa, protagonizada por el gobierno de Calvo Sotelo y su Ministro de Defensa Alberto Oliart, fue breve pero muy intensa: se concentró en la llamada causa 2/81 para el enjuiciamiento de los responsables de la intentona golpista; frente al pronóstico general se consiguió concluir el juicio y las correspondientes condenas con las suficientes dosis de justicia y habilidad como para que no hubiese movimientos en contra de la sentencia. La causa 2/81 por importante que fuera, no agotó la tarea del gobierno Calvo Sotelo en la esfera militar, muy al contrario, se adoptaron en este tiempo medidas trascendentales, sin duda la más importante es la decisión (en mayo 1981) de integrar a España en la Alianza Atlántica, ubicándola así de un modo definitivo en el mundo occidental, lejos de veleidades tercermundistas que todavía anidaban en las mentes de algunos; esta trascendental decisión no solo determina el futuro de España, al incardinarla en Occidente, sino que representa un paso de extraordinaria importancia para la modernización de las Fuerzas Armadas; a partir de ese momento nuestras Fuerzas Armadas abren de par en par sus puertas al exterior y empiezan a relacionarse plenamente con los ejércitos de las demás naciones aliadas. Con anterioridad, especialmente la Armada con el Ejército del Aire, ya tenía tradición de colaboración en ejercicios; ello no solo enriquece profesionalmente a los ejércitos, sino que además les hace ver muy de cerca cuál es la relación de las Fuerzas Armadas aliadas con sus respectivos gobiernos; van constatando así lo trasnochada que queda la institución del Pronunciamiento, comprueban también cómo, en otros países, militares muy parecidos a ellos dependen totalmente de los respectivos gobiernos democráticos, gobiernos de muy distinto signo político.

Otro importante logro es la promulgación de la capital Ley de Dotaciones Presupuestarias de las Fuerzas Armadas (Ley 44/82 de 7 de julio), que muchos quisieron ver como un premio de consolación a estas, pero que fue sobre todo una medida modernizadora, en cuanto permitía el uso de instrumentos de planificación y de contratación hasta entonces inexistentes. Es por tanto la etapa del binomio Calvo Sotelo-Oliart, una etapa, aunque corta, trascendental en nuestro proceso y que, a mi juicio, tampoco ha sido suficientemente reconocida. Esta Ley de Dotaciones permitía que nuestras Fuerzas Armadas empezaran a dotarse de material moderno en los tres ejércitos, aunque tuvo especial repercusión mediática la adquisición de setenta y dos cazas F18, con lo que nuestra fuerza aérea se ponía al nivel de las mejores del mundo, si bien en una escala muy reducida; decisión tomada por el último gobierno de UCD en marzo de 1979, confirmada e implementada por el primer gobierno del PSOE.

La tercera etapa se abre con la victoria socialista de octubre de 1982 y la toma de posesión del Gobierno en el siguiente mes de diciembre. La campaña electoral socialista había girado en torno al eslogan "OTAN, de entrada no", eslogan singularmente hábil por cuanto su ambigüedad lo hacía susceptible de interpretaciones diversas, una de las cuales podía ser "de entrada no ... pero si nos convencen, si"; naturalmente la victoria socialista generó recelos en algunas capitales aliadas, sobre todo en Washington, por lo que muy pronto desfilaron por Madrid importantes mandatarios de la Alianza y entre ellos el Secretario de Defensa de EE. UU. Poco tardó en constatarse que efectivamente la negativa a la Alianza tenía carácter provisional; congelación de la posición española sin entrar en la estructura militar y, por tanto, similar a la posición de Francia y sometida a revisión, que luego se entendió debería someterse a referéndum. Lo cierto es que el binomio Felipe González - Narcís Serra vadeó con gran habilidad el obstáculo del antiamericanismo (previamente atizado por la propia maquinaria del PSOE); esta ambigüedad unida a la reducción de la presencia de tropas norteamericanas en España, generó no pocas incomodidades a ambas partes. Si "por sus frutos los conoceréis" la decisión de ingresar en la Alianza adoptada por Calvo Sotelo y confirmada "post referéndum" por Felipe González el 12 de marzo de 1986', fue un acierto capital; hoy treinta años después, nadie discute el acierto de aquella decisión que incardinó, como decíamos, definitivamente a España en Occidente.

Por lo demás, el citado binomio Felipe González Narcís Serra ejecutó con precisión de relojero el desmontaje del llamado poder militar, no solo con una progresiva y sustancial reducción de efectivos sino también con importantes modificaciones en el despliegue territorial, incluyendo la disminución de capitanías generales. Se acabó con la autonomía de la justicia militar a través de la creación de una sala especial (De lo Militar) en el Tribunal Supremo, que cerraba el arco de la jurisdicción castrense. Asimismo, la enseñanza quedó profundamente modificada. Pero sobre todo, se aseguró la supremacía del llamado poder civil con la consolidación del Ministerio de Defensa (que incluyó dotarle de una nueva sede), a cuyo fren- 
te se puso una mayoría abrumadora de civiles y con la progresiva pérdida de valor de la Junta de Jefes de Estado Mayor, que a partir de entonces tuvo carácter exclusivamente asesor; así como un sinfín de medidas que, como decimos, pusieron fin de una vez y para siempre, a la "autonomía" militar. Sería injusto cerrar esta etapa sin hacer mención a importantes medidas adoptadas por la institución castrense, como fueron la equiparación de salarios con los funcionarios civiles, así como una importante modernización del material, un sustancial incremento de los gastos de I+D y el comienzo de los programas internacionales de cooperación armamentística, entre los que podría ponerse como ejemplo el del Eurofighter, y que sirvieron de contrapunto al conjunto de medidas anteriormente citadas. También es de destacar la participación, cada vez más acusada, de España en operaciones internacionales de paz. La llegada de García Vargas al Ministerio supuso, en este sentido, un bálsamo para las tensiones provocadas por este proceso de desmontaje, quedando para los militares como uno de los mejores ministros de Defensa de la Democracia.

La última etapa del proceso de modernización, sin perjuicio de que siempre es tiempo de nuevas adaptaciones y actualizaciones, fue la protagonizada por el gobierno de José M. a Aznar, en la que caben destacarse dos hitos singulares: la profesionalización de la tropa y marinería, suspendiéndose por tanto el servicio militar obligatorio ${ }^{2}$ (decisión implementada en la segunda legislatura de Aznar bajo la dirección del Ministro Trillo) y la integración en la estructura militar de la Alianza Atlántica, que abría paso a la plena participación y cooperación en el seno de la Alianza sin salvedades ni cortapisas. Una nueva ley de régimen del personal militar e importantes programas de adquisición pueden completar el cuadro de esta etapa, en la que también continuaron ejecutándose, e incluso se incrementaron, las participaciones en operaciones internacionales de paz.

Acabo aquí esta breve excursión por el proceso modernizador, no porque después no se hayan adoptado medidas de gran importancia para la institución militar, sino porque creo que ya han sido medidas relativas a unas Fuerzas Armadas totalmente modernas y modernizadas a las que ya desde entonces se había incorporado la mujer. En efecto, la plena integración en la Alianza Atlántica puso fin al proceso de incorporación, quedando nuestras Fuerzas Armadas insertadas en la Alianza Atlántica de modo definitivo y gozando en ella del general reconocimiento y respeto; por su parte la eliminación del servicio militar obligatorio, aunque produjo efectos negativos -singularmente la disminución del sentimiento de identidad nacional-, equiparó a España con los países de nuestro entorno quienes, antes o después, lo han ido suprimiendo.

\section{DOS NOTAS FINALES SOBRE EL PROCESO DE MODER- NIZACIÓN}

La primera es la de que dicho proceso actuó como bálsamo sobre las tensiones militares de las que hemos hablado antes, bálsamo que permitió una mejor y más feliz cooperación entre civiles y militares; del mismo modo, esta misma modernización jugó un extraordinario papel de lubricante de nuestras relaciones con los aliados una vez realizada la incorporación a la estructura militar, llegándose al caso (antigua Yugoslavia) de que los militares españoles eran los únicos aliados que hacían determinadas misiones combinadas con los norteamericanos.

La segunda es que el proceso no hubiera sido posible sin una gran dosis de consenso; durante todo ese periodo de tiempo y con gobiernos de distinto signo, la política de defensa fue siempre considerada una Política de Estado; sin perjuicio de pequeñas discrepancias, en esta materia fue mucho más lo que unió a los distintos gobiernos que lo que les separó.

Así pues, en aproximadamente un tercio de siglo, las Fuerzas Armadas españolas han protagonizado un cambio en su dimensión, estructura y métodos, sin precedentes en nuestra historia; lo han hecho con una disciplina digna de admiración, que las ha hecho acreedoras del general reconocimiento y comprensión por parte de la sociedad española; aunque la cultura de defensa dista mucho de estar plenamente desarrollada, lo cierto y verdad es que, en los sondeos de opinión, nuestras Fuerzas Armadas son una de las instituciones más valoradas por la ciudadanía, y lo que es aún más importante, unas Fuerzas Armadas que, por su intachable conducta en cuantas intervenciones internacionales han participado, son acreedoras del máximo respeto por parte de los ejércitos aliados. 
1 El 30 de mayo de 1982 España se convirtió en el miembro número 16 de la Alianza Atlántica, tras solicitar formalmente su ingreso el 2 de diciembre anterior y firmar el Protocolo de adhesión el 10 de diciembre de 1981.

\section{BIBLIOGRAFÍA}

Seco Serrano, C. (1984). Militarismo y civilismo en la España Contemporánea. Madrid: Instituto de Estudios Económicos.
2 El 9 de marzo de 2001, el Real Decreto 247/2001 establecía la suspensión del servicio militar obligatorio, más conocido como 'mili', poniendo así fin a una práctica con una historia de más de 200 años en España y abriendo el camino hacia la profesionalización de los Ejércitos en España.

Ley Orgánica 6/1980, de 1 de julio, por la que se regulan los Criterios Básicos de la Defensa. Nacional y la Organización Militar.
Ley 85/1978, de 28 de diciembre, de Reales Ordenanzas para las Fuerzas Armadas. (Vigente hasta el 1 de octubre de 2011). 